\title{
Anelídeos poliquetas da plataforma continental norte do Estado de São Paulo: I - Padrões de densidade e diversidade específica
}

\author{
Paulo Cesar de PAIVA \\ Instituto Oceanográfico da Universidade de São Paulo \\ (Caixa Postal 9075, 01065-970, São Paulo, SP, Brasil)
}

- Abstract: Density and diversity patterns of the polychaetous annelids from the northern continental shelf of São Paulo State, Brazil, were studied. Polychaetous densities were higher in the inner shelf, mainly in the southern transect. Temporal variations were observed at the northern and central transects in the 20 and $35 \mathrm{~m}$ isobaths, with higher summer values. Diversity values were lower in summer and higher during winter in the outer shelf. The inner shelf presented more complex diversity patterns with seasonal fluctuations in lower depths. Muddy sediments presented higher densities; diversity and richness were higher in fine sand sediments. Coarser sediments presented the highest values of evenness but low diversity. The higher diversity values in the shallowest stations were due to the higher number of species and in the deepest stations to the great evenness.

- Resumo: Foram estudados os padrōes de densidade e diversidade específica dos anelídeos poliquetas da plataforma continental norte do Estado de São Paulo. A densidade de poliquetas foi maior na plataforma interna, especialmente na radial Sul. Nas radiais Central e Norte foram observadas variaçōes temporais nas isóbatas de 20 e $35 \mathrm{~m}$, com valores maiores no verão. Os valores de diversidade específica foram preponderantemente mais baixos na plataforma externa, no verāo, e altos, no inverno, enquanto a plataforma interna apresentou padrōes de diversidade mais complexos com flutuaçōes sazonais em baixas profundidades. As densidades foram maiores em sedimentos pelíticos. Areias finas apresentaram valores mais altos de diversidade e riqueza. Sedimentos mais grossos apresentaram eqüitatividades maiores, porém, baixas diversidades. Os valores maiores de diversidade, nas estaçōes mais rasas, foram devidos ao maior número de espécies e nas estaçōes profundas, à maior eqüitatividade.

- Descriptors: Benthic environment, Species diversity, Population density, Temporal variations, Spatial variations, Environmental factors, Sediment-water interface, Polychaeta, Continental shelf, São Paulo State, Brazil.

- Descritores: Ambiente bentônico, Diversidade das espécies, Densidade da população, Variaçōes temporais, Variaçōes espaciais, Fatores ambientais, Interface sedimento-água, Polychaeta, Plataforma continental, Estado de São Paulo, Brasil.

\section{Introdução}

As variaçōes espaciais e temporais da densidade e da diversidade específica estão relacionadas, normalmente, à variabilidade das condiçōes abióticas e bióticas. Fatores como suprimento alimentar, predação, competição, crescimento populacional, perturbaçōes físicas e químicas e outros, que condicionem o ambiente, tendem a alterar a estrutura das comunidades marinhas. As respostas de cada espécie à variabilidade destes fatores se refletem nas taxocenoses em termos de variações da densidade e da diversidade.

A relaçāo da diversidade específica com os fatores ambientais foi a temática de uma série de trabalhos, principalmente no mar profundo (Sanders, 1968; Dayton \& Hessler, 1972; Grassle \& Sanders, 1973) e em costōes rochosos da zona entremarés (Menge \& Sutherland, 1976). A mensuração da diversidade no meio marinho se popularizou, nas décadas de 60 e 70 , como ferramenta na avaliação dos efeitos da poluição em comunidades naturais (Valiela, 1984). 
Variaçōes estruturais (densidade e diversidade) das taxocenoses de anelídeos poliquetas refletem a condição da macrofauna bêntica em geral, já que este grupo taxonômico é um dos mais abundantes em fundos inconsolidados, tanto em número de espécies como de indivíduos, sendo responsável por até $70 \%$ da produtividade da macrofauna em ambientes de plataforma (Knox, 1977).

Os padrões de diversidade de anelídeos poliquetas de plataforma já haviam sidos estudados na regiäo de Ubatuba por Amaral (1980), Lana (1981) e Morgado (1988) na plataforma interna ( $<40 \mathrm{~m}$ de profundidade).

Este estudo visa reconhecer os padrōes de densidade e diversidade e formular hipóteses quanto aos fatores que mais contribuem na estruturação da taxocenose de poliquetas da plataforma continental norte do Estado de São Paulo.

\section{Área de estudo}

A área de estudo (Fig. 1) localiza-se no litoral norte do Estado de São Paulo, entre a Iltha de São Sebastiāo e a Enseada de Picinguaba, sendo limitada pelas latitudes de $23^{\circ} 25^{\prime} \mathrm{S}$ e $24^{\circ} 22^{\prime} \mathrm{S}$ e pelas longitudes de $44^{\circ} 33^{\prime} \mathrm{W}$ e $45^{\circ} 16^{\prime} \mathrm{W}$ e abrangendo a plataforma continental entre 15 e $117 \mathrm{~m}$ de profundidade, perfazendo uma área aproximada de $3800 \mathrm{~km}^{2}$. O fundo da plataforma norte-paulista é coberto, predominantemente, por areias finas e muito finas (Furtado \& Mahiques, 1990). O conteúdo de pelitos é maior ( $>40 \%)$ junto à costa $(20 \mathrm{~m})$, próximo da isóbata de $50 \mathrm{~m}$ e na plataforma externa abaixo dos $100 \mathrm{~m}$. A fraçāo areias prevaleceu mais ao norte onde constituiu entre 80 e $90 \%$ do sedimento.

As condições hidrográficas foram estudadas por Emilsson (1959), Matsuura (1986) e Castro Filho et al. (1987). Três massas de água principais foram reconhecidas: A Água Costeira (AC), a Água Tropical (AT) e a Água Central do Atlântico Sul (ACAS). A AC é restrita a baixas profundidades $(<20 \mathrm{~m})$ no verāo, interagindo com a AT no inverno quando cobrem toda a plataforma interna. A ACAS cobre o fundo da plataforma externa durante todo o ano, penetrando, na plataforma interna, durante o verāo. Assim, a plataforma interna tem uma grande variabilidade intra-anual, apresentando uma termoclina sazonal a $20 \mathrm{~m}$, durante o verão e, praticamente, nenhuma estratificação no inverno.

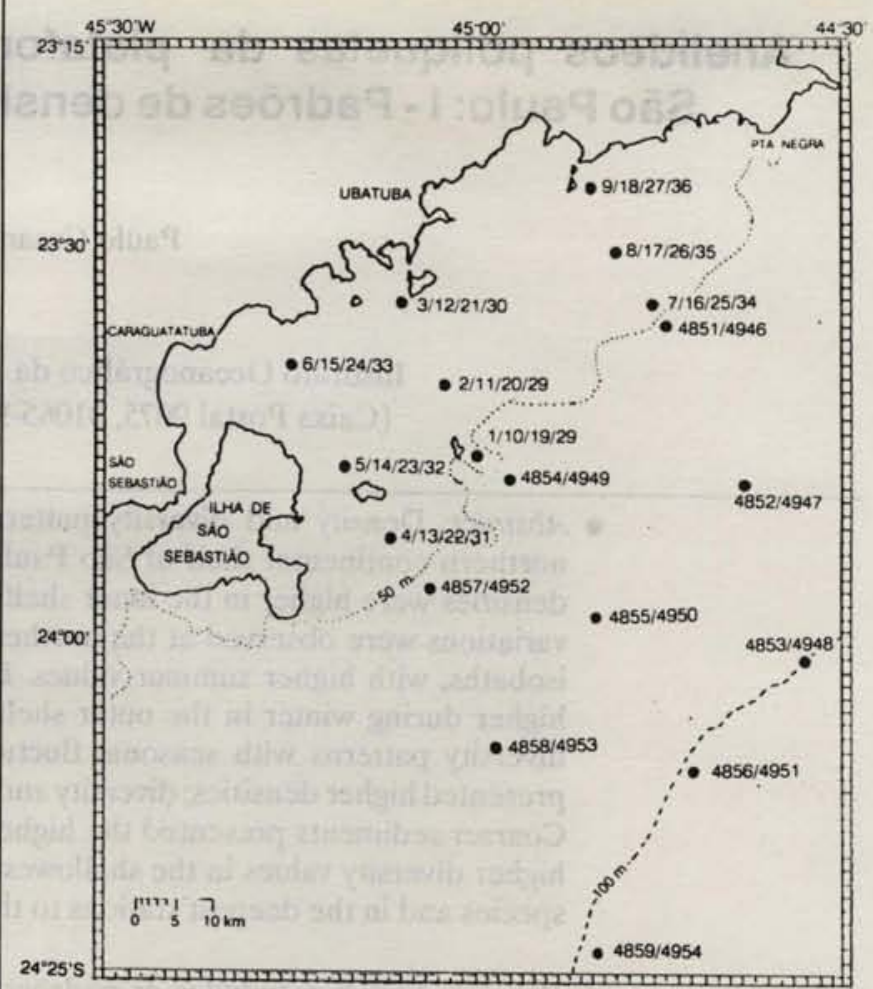

Fig. 1. Áreas de estudo e estações de coleta. Estaçōes 1 a 9 - primavera (10/85); 10 a 18 e 4051 a 4859 - verão (12/85 e 01/86); 19 a 27 - outono $(04 / 86) ; 28$ a 36 e 4946 a 4954 - inverno (07/86).

\section{Material e métodos}

As coletas foram efetuadas em 54 estaçōes oceanográficas distribuídas em dois setores do litoral norte do Estado de São Paulo (Fig. 1), amostradas em diferentes épocas do ano (de outubro de 1985 a julho de 1986). O setor interno (raso) abrange a faixa de 15 a $50 \mathrm{~m}$ de profundidade, onde foram realizadas amostragens sazonais em três radiais: Norte, Central e Sul. Em cada radial foram feitas coletas em três pontos, aproximadamente a 20,35 e $45 \mathrm{~m}$ de profundidade. No setor externo (profundo) foram feitas coletas durante os meses de dezembro (verão) e julho (inverno) em nove pontos, prolongando as radiais do setor interno, nas profundidades de 50, 75 e 100 $\mathrm{m}$, aproximadamente. As coletas do setor interno foram efetuadas com o B/Pq "Veliger II", e as do setor externo com o N/Oc. "Prof. W. Besnard", ambos do Instituto Oceanográfico da Universidade de São Paulo IOUSP.

Nas amostragens de material biológico do subprojeto "Bentos" foi utilizado um pegador-de-fundo, tipo "Van-Veen", de $1 / 10 \mathrm{~m}^{2}$ de área de amostragem. O sedimento, coletado numa única pegada, foi peneirado em 
uma série de três peneiras de malhas 2,1 e $0,5 \mathrm{~mm}$. O material retido nas peneiras foi acondicionado em frascos plásticos e fixado em aldeído fórmico a $4 \%$. Posteriormente, no Laboratório de Biologia Bêntica do Departamento de Oceanografia Biológica do IOUSP, o material foi triado sob microscópio estereoscópico ao nível de grandes grupos taxonômicos (filos, classes ou ordens), a seguir identificados em nível específico e contados.

Em cada estação oceanográfica foram efetuadas coletas de água de fundo, utilizando-se garrafas de Nansen. A temperatura foi medida com termômetros de reversão acoplados às garrafas. Da água coletada foram tomadas amostras para mensuraçāo da salinidade, através de um salinômetro indutivo, e do teor de oxigênio dissolvido, através de titulação pelo método de Winkler (Strickland \& Parsons, 1968). As análises hidrográficas foram efetuadas a bordo e nos laboratórios do Departamento de Oceanografia Física do IOUSP.

Do sedimento coletado pelo pegador-de-fundo, foi tomada uma amostra para análises sedimentológicas. As análises granulométricas foram efetuadas segundo o método de peneiramento e pipetagem descrito em Suguio (1973), obtendo-se os parâmetros estatísticos de Folk \& Ward (1957). Além destes parâmetros, foram utilizadas, as frequências relativas de quatro classes granulométricas: pelitos (argila + silte), areia fina, areia média e areia grossa. $\mathrm{O}$ interesse em separar a areia em três fraçōes se justifica pela seletividade de algumas espécies por fraçōes específicas. Os teores de carbonato biodetrítico do sedimento foram obtidos através de ataque com ácido clorídrico $10 \%$. A análise de teores de matéria orgânica foi feita utilizando-se o processo de oxidaçāo por $\mathrm{H}_{2} \mathrm{O}_{2}$ (Gross, 1971). As análises sedimentológicas foram feitas no Laboratório de Sedimentologia do IOUSP.

A estimativa da densidade de anelídeos poliquetas foi expressa em número de indivíduos por $0,1 \mathrm{~m}^{2}$.

A diversidade específica foi calculada a partir do índice de Shannon-Wiener (Shannon \& Weaver, 1963), derivado da teoria da informação e largamente utilizado em estudos bênticos. Como este foi calculado utilizando-se logarítmo na base 2 , a diversidade foi expressa em bits/indivíduo. A eqüitatividade foi calculada através do índice de Pielou (1975).

Para a expressão do grau de dependência entre a densidade, riqueza, diversidade e eqüitatividade com as variáveis ambientais, foi efetuada uma análise de "regressāo múltipla passo a passo" (Legendre \& Legendre,
1983), onde as variáveis independentes que melhor explicaram os modelos de regressão múltipla foram selecionadas ao nível de $5 \%$ de significância. Para a análise de regressão múltipla, os valores das variáveis: profundidade, temperatura, salinidade, oxigênio dissolvido e riqueza de espécies foram normalizados através da tranformação em raiz quadrada. Para a normalizaçāo da densidade foi extraída a raiz da raiz quadrada e para os dados expressos em porcentagem (matéria orgânica, carbonato biodetrítico, areia fina e pelitos) foi feita a transformaçāo arcseno. As demais variáveis (diversidade, eqüitatividade e coeficiente de selecionamento) não foram transformadas, pois seus cálculos já envolvem uma logaritmizaçāo.

\section{Resultados}

A Tabela 1 fornece os parâmetros das variáveis ambientais, para cada uma das estaçōes de amostragem, nos setores interno e externo durante o período de coleta $(10 / 85$ a 07/86) e a Figura 1 fornece a localização e a época de amostragem das estaçōes de coleta.

Foi identificado um total de 166 espécies de anelídeos poliquetas pertencentes a 38 famílias. As espécies e suas distribuiçōes nas estaçōes de coleta constam das Tabelas 2 (plataforma interna) e 3 (plataforma externa).

A Tabela 4 fornece os valores da densidade, riqueza, diversidade e eqüitatividade, para cada uma das estações de coleta.

$\mathrm{Na}$ plataforma interna, a densidade expressa em número de indivíduos por $0,1 \mathrm{~m}^{2}$, foi maior na radial Sul durante todo o ano (exceto na primavera). As densidades maiores nesta radial se deveram, principalmente, à abundância de espécies da superfície do sedimento como Spiophanes missionensis, Cirrophorus americanus, Cirratulus filiformis, Magelona posterolongata. $\mathrm{Na}$ radial Central, os valores foram intermediários $\left(20-70\right.$ ind $\left./ 0,1 \mathrm{~m}^{2}\right)$ durante todo o ano, exceto pela isóbata de $20 \mathrm{~m}$ onde ocorreu uma flutuaçāo temporal marcante, atingindo um pico máximo de densidade no verão (932 ind.) devido, principalmente, à grande abundância de Hydroides plateni, Chone insularis e Polydora socialis. Na radial Norte, as densidades foram maiores também no período de verão (>50 ind).

Na plataforma externa, ao contrário da interna, as densidades foram maiores no inverno, embora o pico maior tenha ocorrido numa estação de verão (Est. 4851, radial Norte, isóbata de $50 \mathrm{~m}$ ). O modelo de regressão múltipla para densidade selecionou apenas a variável temperatura (positivamente). 
Tabela 1. Datas das coletas e dados ambientais das estações oceanográficas (profundidade, temperatura, salinidade, oxigênio dissolvido, matéria orgânica, carbonato biodetrítico, pelitos, areia grossa, areia média, areia fina e coeficiente do selecionamento). $\mathrm{P}=$ primavera, $\mathrm{V}=$ verão, $\mathrm{O}=$ outono, $\mathrm{I}=$ inverno, $\mathrm{S}=$ radial Sul, $\mathrm{C}=$ radial Central, $\mathrm{N}=$ radial Norte.

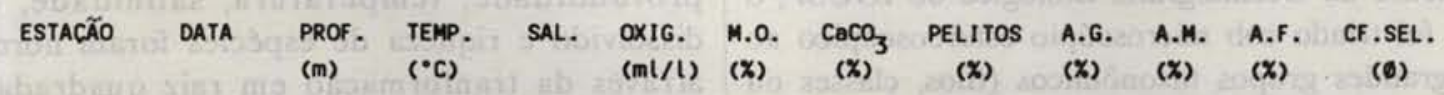

\begin{tabular}{|c|c|c|c|c|c|c|c|c|c|c|c|c|c|c|}
\hline & PC & $26-10-85$ & 46 & 16,54 & 35,58 & 0,37 & 2,1 & 40,5 & 16,46 & 26,0 & 14,2 & 44,4 & 2,18 & \\
\hline & PC & $26-10-85$ & 35 & 16,79 & 35,61 & 3,58 & 0,7 & 36,1 & 3,22 & 2,2 & 3,4 & 91,2 & 0,43 & \\
\hline & PC & $26-10-85$ & 19 & 22,21 & 35,47 & 4,99 & 2,3 & 25,1 & 25,93 & 5,2 & 13,4 & 55,6 & 1,94 & $1 n^{2}=$ \\
\hline & PS & $27-10-85$ & 40 & 17,41 & 35,56 & 3,48 & 1,3 & 17,8 & 20,42 & 7,1 & 10,0 & 62,5 & 1,88 & sh \\
\hline & PS & $27-10-85$ & 32 & 19,44 & 35,52 & 4,21 & 2,5 & 15,1 & 26,12 & 11,8 & 12,8 & 2,1 & 2,4 & \\
\hline & PS & $27-10-85$ & 15 & 21,30 & 35,54 & 4,95 & 2,1 & 15,5 & 42,6 & 2,4 & 4,6 & 50,4 & 1,79 & \\
\hline & PN & $28-10-85$ & 43 & 15,58 & 35,56 & 4,09 & 2,3 & 12,3 & 11,56 & 3,1 & 3,1 & 82,3 & 1,07 & \\
\hline & PN & $28-10-85$ & 38 & 16,40 & 35,63 & 3,22 & 0,1 & 57,3 & 6,38 & 47,8 & 38,3 & 10,4 & 1,06 & \\
\hline & PN & $28-10-85$ & 17 & 20,48 & 35,56 & 4,49 & 2,8 & 16,0 & 15,11 & 5,9 & 11,8 & 67,4 & 1,6 & \\
\hline 10 & vc & $20-01-86$ & 45 & 15,66 & 35,60 & 4,75 & 1,4 & 20,9 & 41,23 & 21,2 & 7,6 & 31,9 & 3,17 & \\
\hline 11 & vc & $20-01-86$ & 36 & 15,77 & 35,54 & 4,44 & 0,7 & 29,2 & 7.23 & 5,5 & 3,5 & 84,5 & 0,77 & \\
\hline 12 & & $20-01-86$ & 21 & 23,54 & 35,47 & 2,65 & 1,7 & 39,5 & 15,57 & 12,2 & 30,8 & 41,7 & 1,66 & \\
\hline 13 & & $21-01-86$ & 38 & 15,62 & 35,48 & 3,10 & 1,4 & 14,0 & 17,91 & 2,3 & 3,7 &, 2 & 1,05 & \\
\hline 14 & vs & $21-01-86$ & 35 & 15,70 & 35,44 & & 1,2 & 17,0 & 14,01 & 7,2 & 8,3 & 70,7 & 1,42 & \\
\hline 15 & vs & $21-01-86$ & 20 & 16,27 & 35,45 & 3,07 & 0,5 & 15,8 & 24,82 & 4,5 & 4,5 & 66,6 & 1,58 & \\
\hline 16 & W & $22-01-86$ & 47 & 15,64 & 35,53 & 3,67 & 1,9 & 12,9 & 10,96 & 1,5 & 4,6 & 83,0 & 0,98 & \\
\hline 17 & W & $22-01-86$ & 44 & 15,83 & 35,56 & 3,89 & 1,6 & 23,0 & 8,25 & 5,4 & 3,7 & 9 & 1 & \\
\hline 18 & W & $22-01-86$ & 18 & 18,41 & 35,42 & 3,60 & 0,5 & 14,7 & 10 & 2,0 & 3,8 & 83,7 & 1 & \\
\hline 19 & $\propto$ & $20-04-86$ & 46 & 18,69 & 35,77 & 3,69 & 2,7 & 34,6 & 28,59 & 16,2 & 14,2 & 42,6 & 2,35 & \\
\hline 20 & $\propto$ & $20-04-86$ & 35 & 19,40 & 35,87 & 3,66 & 1,7 & 11,9 & 9,38 & 0,5 & 0,4 & 89,8 & 0,46 & \\
\hline 21 & $\propto$ & $18-04-86$ & 20 & 25,73 & 35,42 & 4,52 & 1.4 & 57,2 & 11 & 51,3 & 32,1 & 5,8 & 1,34 & \\
\hline 22 & os & $19-04-86$ & 40 & 25,33 & 35,42 & 4,39 & 1,1 & 20,0 & 16,33 & 2,8 & 13,3 & 67,6 & 1,35 & \\
\hline 23 & os & $19-04-86$ & 36 & 25,43 & 35,48 & 4,34 & 1, & 7,8 & 1 & 4,5 & 11,2 & 75,2 & 1,16 & \\
\hline 24 & os & $18-04-86$ & 16 & 5,41 & 35,42 & 4,40 & 2,7 & 15 & 42 , & 0,2 & 1,2 & 55,6 & 1 & \\
\hline 25 & ON & $20-04-86$ & 45 & 18,24 & 35,92 & 4,01 & 2,2 & 17,0 & 14,08 & 8,2 & 6,6 & 71,2 & 1,37 & \\
\hline 26 & ON & $21-04-86$ & 42 & 18,69 & 36,08 & 3,51 & 0,3 & 19,0 & 7,95 & 5,1 & 4,7 & 82,3 & 0,92 & \\
\hline 27 & ON & $21-04-86$ & 21 & 24,78 & 35,61 & 3,19 & 0,8 & 12,0 & 9,11 & 2,2 & 8,4 & 80 & 1,03 & \\
\hline 28 & IC & $09-07-86$ & 48 & 22,50 & 35,80 & 4,61 & 2,6 & 12,4 & 36,43 & 12,1 & 13,0 & 39,0 & 2,44 & \\
\hline 29 & IC & $09-07-86$ & 35 & 22,42 & 35,87 & 4,87 & 1,6 & 12,3 & 7,41 & 0,3 & 0,6 & 91,7 & 0,48 & \\
\hline 30 & IC & $09-07-86$ & 22 & 22,34 & 35,81 & 4,39 & 3,9 & 8,9 & 34, & 8,8 & 8,5 & 48 & 2,09 & \\
\hline 31 & IS & $10-07-86$ & 38 & 20,00 & 35,85 & 4,54 & 2,3 & 5 , & 19,75 & 0,4 & 1,4 & 78,8 & 1,08 & \\
\hline 32 & Is & $10-07-86$ & 35 & 22,20 & 5,83 & 4,52 & 2,3 & 9, & 20,87 & 4,1 & 8,6 & 66,4 & 1,56 & \\
\hline 33 & IS & $10-07-86$ & 17 & 22,22 & 35,76 & 5,13 & 3,8 & 9,5 & 4 & 1,7 & 1,5 & 44,4 & 1,86 & \\
\hline 34 & IN & $11-07-86$ & 48 & 17,93 & 35,73 & 3,20 & 2,2 & 6,7 & 18,98 & 0,3 & 1,0 & 79,7 & 1,17 & \\
\hline 35 & IN & $11-07-86$ & 41 & 18,94 & 35,77 & 3,18 & 0,2 & 25,5 & 0,04 & 19,4 & 43,7 & 36,9 & 0,89 & \\
\hline 36 & IN & $11-07-86$ & 24 & 22,33 & 35,82 & 4,57 & 2,3 & 8,7 & 38,21 & 10,2 & 10,9 & 41,2 & 2,47 & \\
\hline 851 & WN & $16-12-85$ & 48 & 14,45 & 35,42 & 2,98 & 1,4 & 12,5 & 4,05 & 0,3 & 1,1 & 94 & 0,35 & \\
\hline 852 & W & $16-12-85$ & 70 & 15,03 & 35,51 & 3,96 & 0,4 & 17,7 & 3,72 & 32,4 & 13,5 & 50,7 & 1,24 & \\
\hline 853 & WN & $16-12-85$ & 104 & 13,73 & 35,34 & 4,63 & 1,6 & 21,5 & 10,24 & 13,1 & 15,2 & 61,4 & 1,74 & \\
\hline 854 & ve & $17-12-85$ & 47 & 14,87 & 35,47 & 3,68 & 2,2 & 12,3 & 30,88 & 0,1 & 1,1 & 67 & 1,56 & \\
\hline 355 & ve & $17-12-85$ & 73 & 14,33 & 55,43 & 4,42 & 0,7 & 30,5 & 7,4 & 5,6 & 5,4 & 81 & 1,01 & \\
\hline 856 & ve & $17-12-85$ & 116 & 14,13 & 35,40 & 4,48 & 0,8 & 28,3 & 23,24 & 6,6 & 8,8 & 61,5 & 2,17 & \\
\hline 857 & vs & $18-12-85$ & 48 & 15,45 & 35,55 & 4,01 & 0,1 & 41,3 & 10,78 & 0,7 & 8,8 & 79,8 & 1,31 & \\
\hline 858 & vs & $18 \cdot 12 \cdot 85$ & 76 & 14,43 & 5,44 & 4,58 & 3,0 & 17,8 & 32,39 & 3,2 & 5,1 & 59 & 2,5 & \\
\hline 859 & vs & $18 \cdot 12 \cdot 85$ & 102 & 14,81 & 35,49 & 4,52 & 1,3 & 51,7 & 21,97 & 14,7 & 4,1 & 59,4 & 2,25 & \\
\hline 946 & IN & $26-07-86$ & 50 & 21,80 & 35,74 & 4,59 & 0,9 & 5,5 & 13,51 & 1,1 & 2,6 & 82,8 & 0,86 & \\
\hline 947 & IN & $26-07-86$ & $\pi$ & 16,72 & 35,69 & 3,90 & 1,1 & 13,4 & 12,56 & 18,8 & 9,8 & 59,5 & 1,68 & \\
\hline 948 & IN & $26-07-86$ & 108 & 15,61 & 35,54 & 4,05 & 0,3 & 8,6 & 13,49 & 24,4 & 19,4 & 42,8 & 1,7 & \\
\hline 949 & IC & $27-07-86$ & 50 & 21,38 & 35.93 & 8 & 1,5 & 7,4 & 33,45 & 1,0 & 3,2 & 62,3 & 1,48 & \\
\hline 50 & IC & $27-07-86$ & 75 & 17,37 & 35,77 & 3,88 & 1,5 & 15,9 & 0,01 & 3,6 & 5,9 & 90,5 & 0,54 & \\
\hline 51 & IC & $27-07-86$ & 117 & 15,81 & 35,63 & 4,00 & 1,4 & 11,4 & 19,95 & 33,4 & 7,9 & 40,5 & 2,17 & \\
\hline 52 & IS & $28-07-86$ & 48 & 21,92 & 35,93 & 4,26 & 2,0 & 5,5 & 19,04 & 0,4 & 2,0 & 78,7 & 1,05 & \\
\hline 53 & is & $28-07-86$ & 79 & 18,66 & 35,90 & 3,24 & $\cdot$ & $\cdot$ & $\cdot$ & . & - & $\cdot$ & - & \\
\hline 54 & IS & $28-07-86$ & 101 & 18,13 & 36,06 & 4,36 & 0,8 & 25,9 & 12,65 & 20,4 & 4,3 & 62,8 & 1,76 & \\
\hline
\end{tabular}


Tabela 2. Densidade das espécies (indivíduos $/ 0,1 \mathrm{~m}^{2}$ ) de poliquetas encontrados nas estações de coleta da plataforma interna

\section{Aphroditella alta}

Eunoe sernata

Harmothoe hunulata

Harmothoe sp

Lepidasthenia virens

Lepidonotus sp

Sthenelais sp

Sthenolepis cf. oculate

Pholoe synophthalmica

Linophenus ambigua

Ancitides mucosa

Eteone heteropoda

Eulalia cf. bilineata

Gyptis callithrix

Ophiodromus pugetensis

Podarke of. obscura

Ancystrosyllis jonesi

Cabira incerta

Parandalia tricuspis

Sigambra grubii

Enogone arenosa

Enogone dispar

Langerthansia comuta

Pionosyllis pectinata

Typosyllis hyalina

Ceratocephale oculata

Neanthes brusce

Neanthes of. succinea

Nereis broa

Nereis sp

Nicon sp

veresiolowe - ño idant if.

Aglaophamus juvenalis

Inemonephtys palpate

Nephtys acrochaeta

Nephtys simoni

Nephrys spp

Glycera americana

Hemipodus olivieri

Hemipodus rotundus

Glycinde multidens

Goniada bnunnea

Goniada maculata

Goniadides canolinae

Ophyoglycera enimia

COWINDIDAE - nẽo identif.

Diopatra cf. cupres

Diopatra tridentata

Kinbergonuphis difficilis

Kinbergonuphis orensanzi

Kinbergonuphis spp

Mooreonuphis intermedia

Nothria benthophyla

Lumbrinerides gesae

Lumbrineridopsis mucronata

Lumbrineris cingulata

Lumbrineris janeirensis

Lumbrineris tetraura

Ninoe brasiliensis

Arabella inicolor

Arabella sp

Orilonereis fihum

Orilonereis mulleri

Notocimus lonum

Schistoneringos nudolphi

Leitoscoloplos cf. fragilis

Phylo felin

Scoloplos (S.) treadwelli

Scoloplos (Scoloplos) spp

Scoloplos (Leodamas) dubia

Scoloplos (Leodamas) ohlini
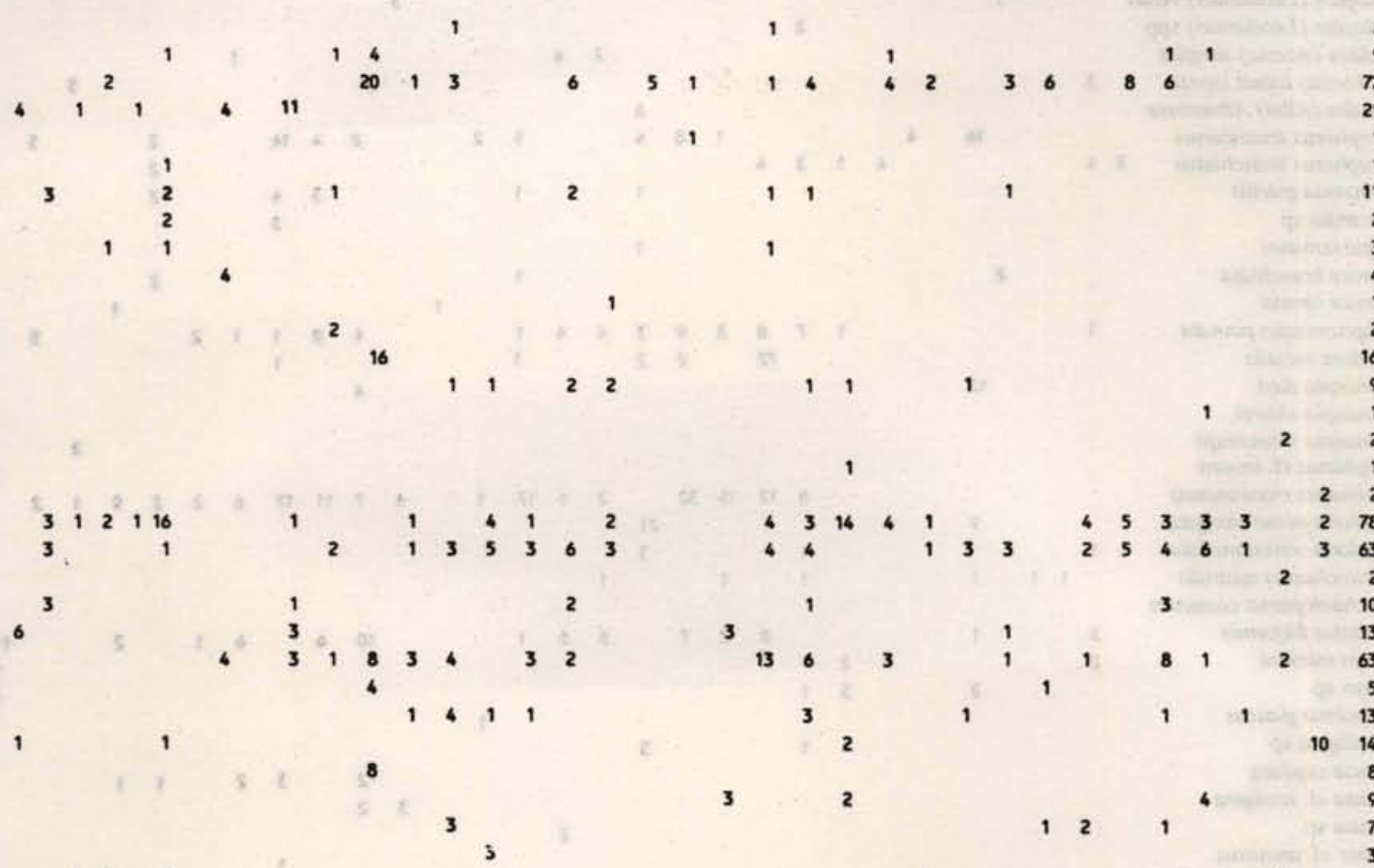

3

136

3

3

2

213

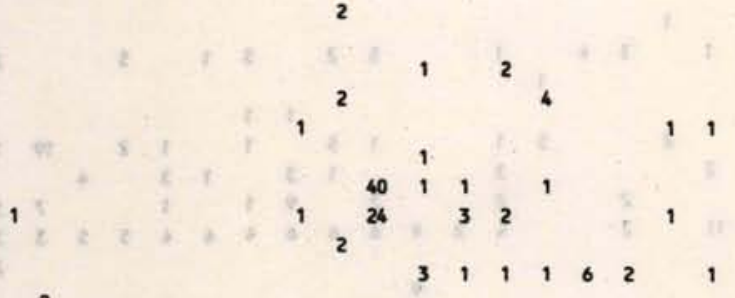

$?$

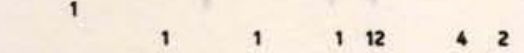

(4) 120

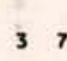

43

$\begin{array}{lllllll}1 & & & 1 & & & \\ 6 & 1 & 2 & 4 & 3 & 12 & 1\end{array}$ 4122

a
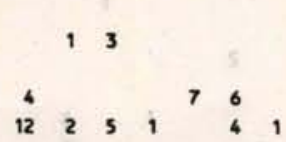

22

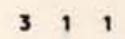

221

\section{2}

72 
Tabela 2. Continuação

ESPECIES/ESTAÇ̌̃SS $\begin{array}{lllllllllllllllllllllllllllllllllllllllllll}1 & 2 & 3 & 4 & 5 & 6 & 7 & 8 & 9 & 10 & 11 & 12 & 13 & 14 & 15 & 16 & 17 & 18 & 19 & 20 & 21 & 22 & 23 & 26 & 25 & 26 & 27 & 28 & 29 & 30 & 31 & 32 & 33 & 34 & 35 & 36 & \text { TOTAL }\end{array}$

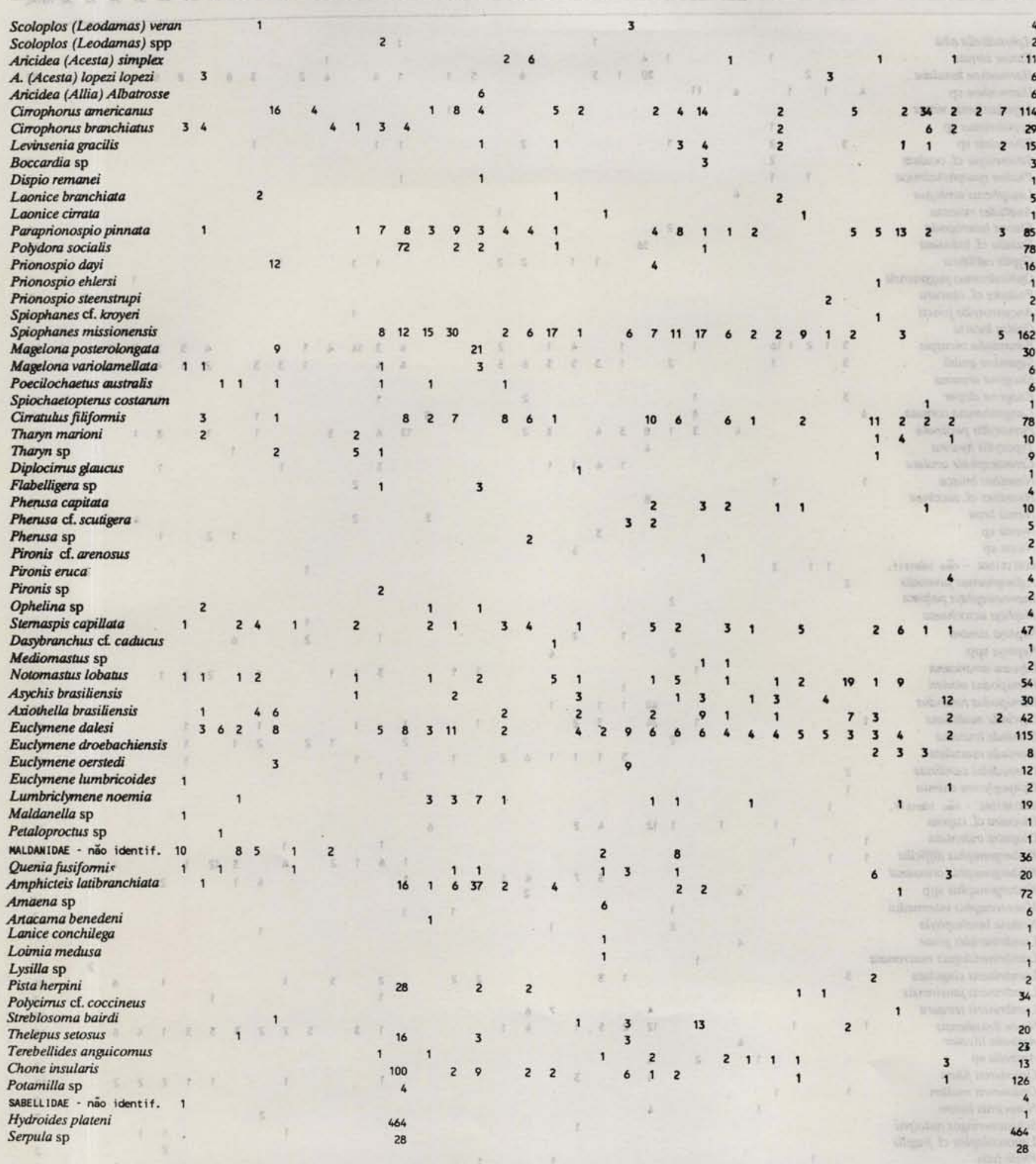

TOTAL 
Tabeia 3. Densidade das espécies (indivíduos $/ 0,1 \mathrm{~m}^{2}$ ) de poliquetos encontrados nas estações de coleta da plataforma interna

ESPECIES/ESTACOES $\quad 4851485248534854 \quad 48554856485740584059494649474948494949504951495249534954$ TOTAL

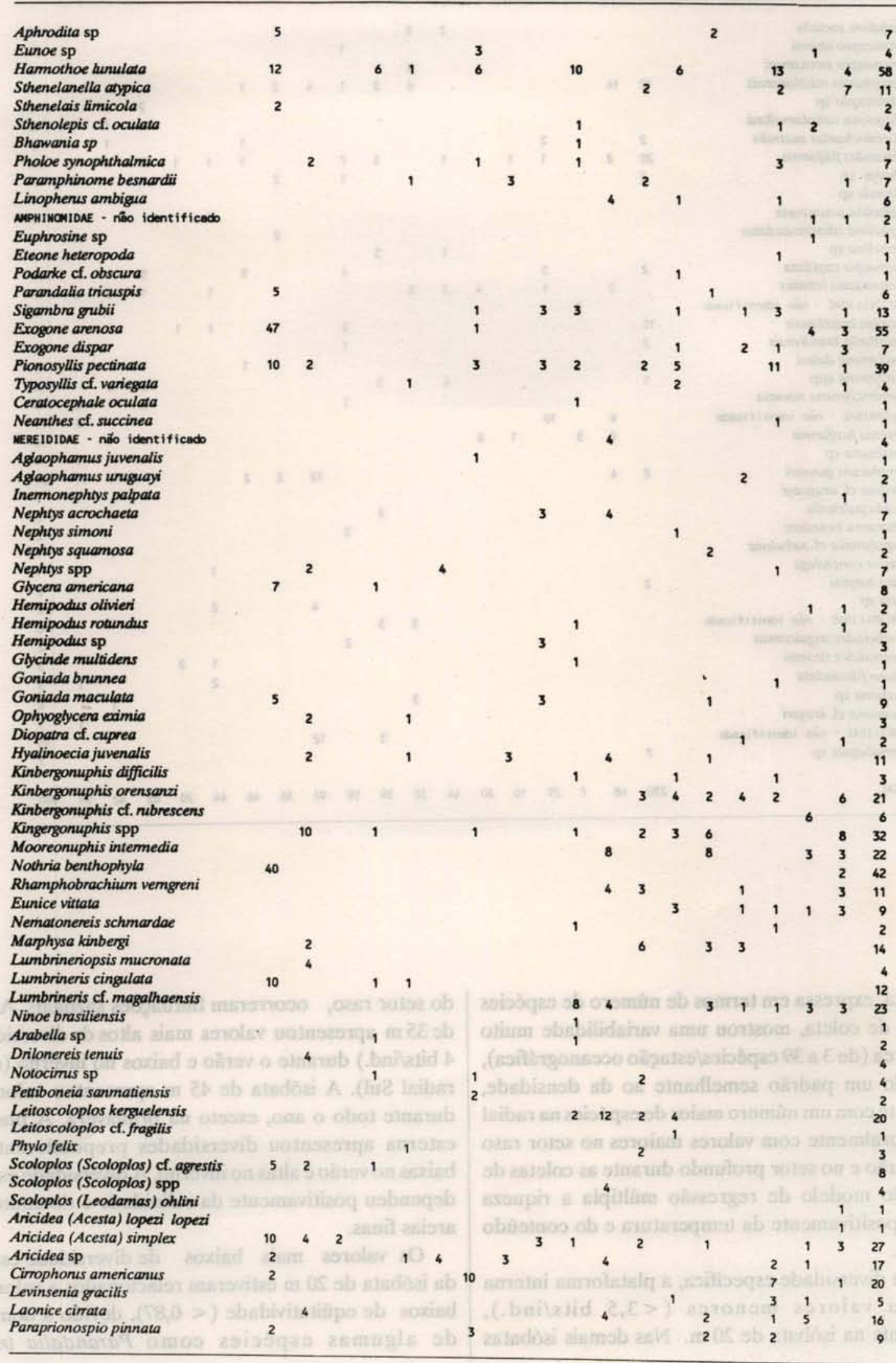


Tabela 3. Continuação

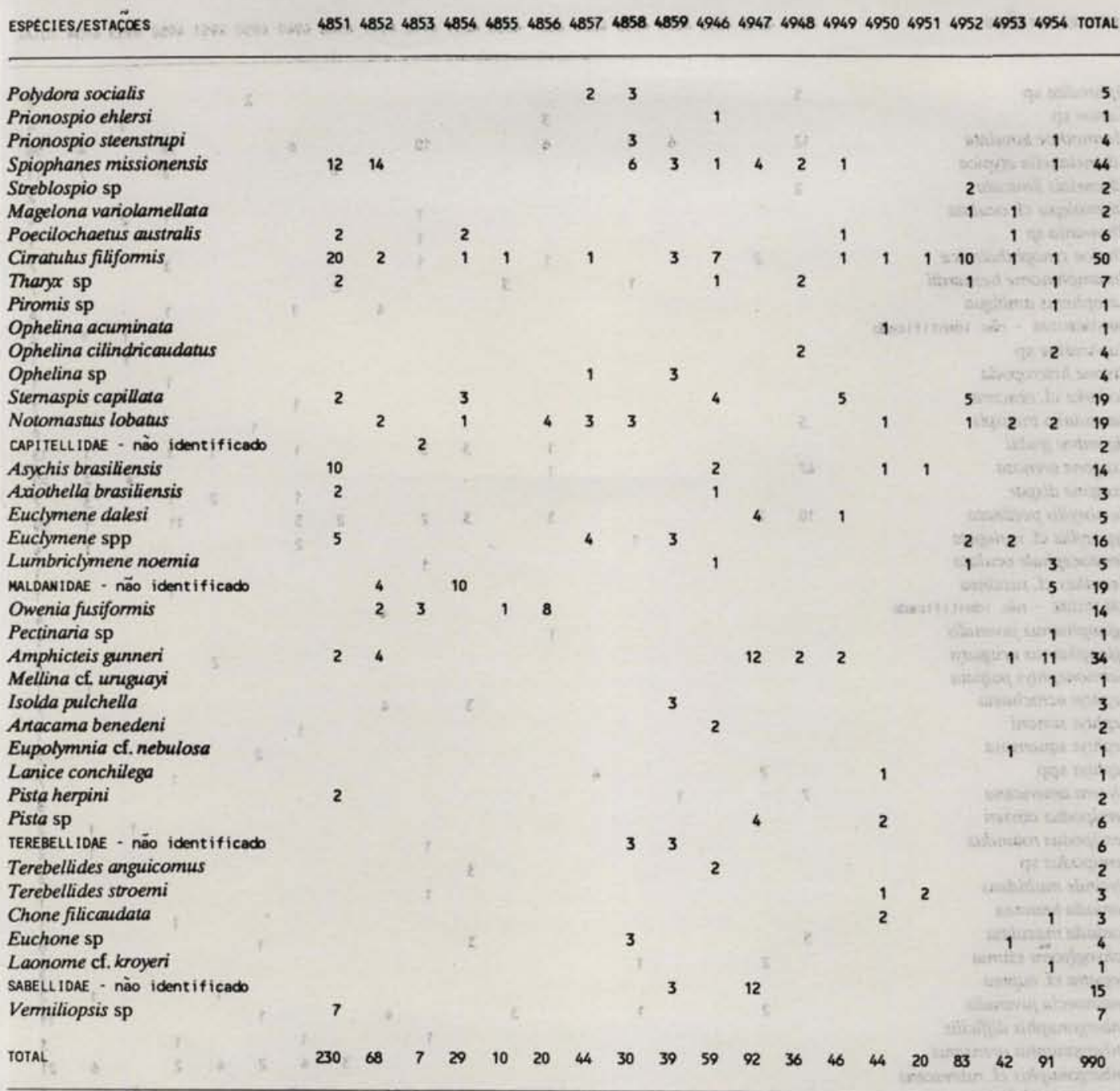

A riqueza, expressa em termos de número de espécies por estação de coleta, mostrou uma variabilidade muito grande na área (de 3 a 39 espécies/estação oceanográfica), apresentando um padrão semelhante ao da densidade, espacialmente com um número maior de espécies na radial Sul e temporalmente com valores maiores no setor raso durante o verão e no setor profundo durante as coletas de inverno. Pelo modelo de regressão múltipla a riqueza dependeu positivamente da temperatura e do conteúdo de areia fina.

Quanto à diversidade específica, a plataforma interna apresentou valores menores ( $<3,5$ bits/ind.), principalmente na isóbata de $20 \mathrm{~m}$. Nas demais isóbatas do setor raso, ocorreram flutuaçōes sazonais. A isóbata de $35 \mathrm{~m}$ apresentou valores mais altos de diversidade (> 4 bits/ind.) durante o verão e baixos no inverno (exceto a radial Sul). A isóbata de $45 \mathrm{~m}$ apresentou valores altos durante todo o ano, exceto na primavera. A plataforma externa apresentou diversidades preponderantemente baixas no verāo e altas no inverno. A diversidade específica dependeu positivamente da salinidade e do conteúdo de areias finas.

Os valores mais baixos de diversidade específica da isóbata de $20 \mathrm{~m}$ estiveram relacionados a valores mais baixos de eqüitatividade $(<0,87)$, devido à dominância de algumas espécies como Parandalia tricuspis, 
Tabela 4. Valores de densidade (indivíduos $/ 0,1 \mathrm{~m}^{2}$ ). Riqueza (número de espécies), índices de diversidade (bits/ind.) e eqüitatividade das estações de coleta. $\mathrm{P}=$ primavera, $\mathrm{V}=$ verão, $\mathrm{O}=$ outono, $\mathrm{I}=$ inverno, $\mathrm{S}=$ radial Sul, $\mathrm{C}=$ radial Central, $\mathrm{N}=$ radial norte

\begin{tabular}{|c|c|c|c|c|c|}
\hline ESTAC & CAO & N & s & $\mathrm{H}^{\prime}$ & E \\
\hline 1 & PC & 35 & 16 & 3,37 & 0,84 \\
\hline & PC & 44 & 20 & 4,23 & 0,97 \\
\hline & PC & 15 & 10 & 2,87 & 0,86 \\
\hline & PS & 23 & 12 & 3,13 & 0,87 \\
\hline & PS & 22 & 10 & 3,02 & 0,90 \\
\hline & PS & 92 & 23 & 3,73 & 0,82 \\
\hline 7 & PN & 5 & 5 & 2,30 & 1,00 \\
\hline & PN & 28 & 7 & 2,80 & 1,00 \\
\hline & PN & 7 & 3 & 1,37 & 0,86 \\
\hline 10 & ve & 37 & 16 & 3,74 & 0,93 \\
\hline 11 & ve & 49 & 22 & 4,06 & 0,91 \\
\hline 12 & ve & 932 & 27 & 2,99 & 0,62 \\
\hline 13 & vs & 51 & 23 & 3,93 & 0,86 \\
\hline 14 & vs & 121 & 26 & 4,03 & 0,85 \\
\hline 15 & vs & 142 & 31 & 3.95 & 0,79 \\
\hline 16 & w & 53 & 21 & 4,05 & 0,92 \\
\hline 17 & Ww & 76 & 20 & 4,16 & 0,96 \\
\hline 18 & $w$ & 52 & 19 & 3,51 & 0,82 \\
\hline 19 & $\propto$ & 26 & 14 & 3,53 & 0,92 \\
\hline 20 & $\propto$ & 21 & 13 & 3,36 & 0,90 \\
\hline 21 & $\propto$ & 57 & 12 & 3,43 & 0,95 \\
\hline 22 & os & 82 & 27 & 4,23 & 0,88 \\
\hline 23 & os & 103 & 32 & 4,59 & 0,91 \\
\hline 24 & os & 107 & 25 & 3,90 & 0,83 \\
\hline 25 & ON & 51 & 21 & 4,06 & 0,92 \\
\hline 26 & ON & 28 & 16 & 3,74 & 0,93 \\
\hline 27 & ON & 36 & 19 & 4,07 & 0,95 \\
\hline 28 & IC & 51 & 23 & 4,11 & 0,90 \\
\hline 29 & IC & 38 & 14 & 3,48 & 0,91 \\
\hline 30 & IC & 71 & 16 & 3,41 & 0,85 \\
\hline 31 & IS & 69 & 25 & 4,15 & 0,89 \\
\hline 32 & is & 89 & 30 & 4,40 & 0,89 \\
\hline 33 & is & 80 & 21 & 3,29 & 0,74 \\
\hline 34 & IN & 62 & 23 & 4,07 & 0,89 \\
\hline 35 & IN & 16 & 8 & 3,00 & 1,00 \\
\hline 36 & IN & 36 & 9 & 2,89 & 0,91 \\
\hline 4851 & W & 230 & 26 & 3,94 & 0,83 \\
\hline 4852 & WN & 68 & 18 & 3,81 & 0,91 \\
\hline 4853 & W & 7 & 3 & 1,55 & 0,97 \\
\hline 4854 & ve & 29 & 12 & 2,94 & 0,82 \\
\hline 4855 & ve & 11 & 11 & 3,45 & 0,99 \\
\hline 4856 & ve & 20 & 4 & 1,92 & 0,96 \\
\hline 4857 & vs & 44 & 17 & 3,64 & 0,89 \\
\hline 4858 & vs & 30 & 9 & 3,12 & 0,98 \\
\hline 4859 & vs & 45 & 15 & 3,90 & 0,99 \\
\hline 4946 & IN & 59 & 27 & 4.19 & 0,88 \\
\hline 4947 & IN & 92 & 16 & 3,82 & 0,95 \\
\hline 4948 & IN & 36 & 15 & 3,80 & 0,97 \\
\hline 4949 & IC & 46 & 20 & 3,97 & 0,91 \\
\hline 4950 & IC & 44 & 21 & 4,02 & 0,91 \\
\hline 4951 & IC & 20 & 12 & 3,38 & 0,94 \\
\hline 4952 & is & 83 & 30 & 4,24 & 0,86 \\
\hline 4953 & Is & 42 & 23 & 4,18 & 0,92 \\
\hline 4954 & Is & 92 & 39 & 4,80 & 0,90 \\
\hline
\end{tabular}

Cirrophorus americanus, Spiophanes missionensis e Polydora socialis. Por sua vez, as diversidades mais baixas de algumas estaçōes do setor profundo estiveram relacionadas a valores altos de eqüitatividade devidas ao baixo número de espécies. A eqüitatividade dependeu, positivamente, do conteúdo de oxigênio dissolvido, do coeficiente de selecionamento e negativamente do conteúdo de pelitos.

\section{Discussão}

As maiores densidades e o maior número de espécies de poliquetas na radial Sul, ao longo do ano, devêm-se, provavelmente, à estabilidade do sedimento e ao seu conteúdo de pelitos, nesta que é considerada a maior zona de deposição na área (Furtado \& Mahiques, 1990). Sedimentos pelíticos, em geral, permitem o estabelecimento de um número maior de espécies da infauna como depositívoros e carnívoros de subsuperfície num estágio sucessional avançado (Rhoads \& Germano, 1982). Flutuaçōes na densidade e riqueza, nas radiais Central e Norte, podem estar relacionadas à instabilidade ambiental causada pela maior incidência de ondas no período de inverno e pela estratificação de massas de água no período de verão (Castro Filho et al., 1987) levando a um florescimento de formas de superfície (Probert, 1984).

Na plataforma externa, as diferenças encontradas quanto ao número de espécies entre verāo e inverno, não devem estar associadas a estabilidade ambiental pois, nas isóbatas de 75 e $100 \mathrm{~m}$, as condiçōes ambientais devem ser constantes ao longo do ano em função do padrão de ondas incidentes na costa de Sāo Paulo (Tessler, 1988) que afeta, principalmente, a plataforma interna. As diferenças sāo, provavelmente, devidas à amostragem em pontos diferentes nos dois períodos. Os pontos amostrados no verāo, de natureza pelítica, seriam viáveis para apenas algumas espécies características com o Hyalinoecia juvenalis, Paramphinome besnardi e Prionospio steenstrupi.

Nāo é conclusiva a dependência da densidade em relação à temperatura. No entanto, altas temperaturas no fundo estâo relacionadas à presença da AC (Água Costeira), ligadas, portanto, a fundos rasos, embora ainda nāo possa ser descartada a influência térmica na produtividade dos poliquetas.

Nos fundos pelíticos, a riqueza de espécies nāo encontrou correspondência na diversidade devido à dominância de Cirrophorus americanus, Spiophanes missionensis, Magelona posterolongata. Por outro lado, a alta eqüitatividade registrada nos fundos arenosos (correlação negativa com pelitos) mal selecionados, ou seja, mistura de areias finas, médias e grossas, correspondeu a baixos valores de diversidade, indicando que apenas algumas espécies, como: 
Langerhansia cornuta, Eunice vittata, Sthenellanela atypica, Linopherus ambigua, Leitoscoloplos kerguelensis e Scoloplos agrestis se adequam bem a este tipo de fundo, geralmente instável (de alta mobilidade).

Embora normalmente a diversidade seja maior em sedimentos mais heterogêneos, devido a maior disponibilidade de nichos exploráveis (Gray, 1981; Lana, 1982; Fresi et al., 1983), tal tendência não foi observada neste estudo. $\mathrm{Na}$ realidade, as areias finas melhores selecionadas (a variável coeficiente de selecionamento não participou do modelo de regressão por ser dependente do conteúdo de areia fina) apresentaram maior número de espécies, que se refletiu nos valores mais altos de diversidade neste tipo de fundo.

A dependência da riqueza e da diversidade em relação à porcentagem de areia fina é conhecida da literatura nas taxocenoses de areias finas de Picard (1965 apud Fresi et al., 1983; Massé, 1972; Gambi \& Fresi, 1981) no Mediterrâneo e nas costas atlântica (Weston, 1988) e pacífica da América do Norte (Hyland et al. 1991). Sedimentos arenosos são mais permeáveis, permitindo uma maior penetraçâo de oxigênio (Weston, op. cit.), e, portanto, ampliando a camada de sedimento a ser explorada pela macrofauna. Não obstante, em estudos anteriores realizados na plataforma interna da região, Lana (1981) e Morgado (1988) constataram, pelo contrário, uma pobreza em poliquetas nas areias finas bem selecionadas. Uma hipótese plausível é a de que, os fundos arenosos de baixas profundidades, são mais instáveis que os de regiōes mais profundas devido a maior intensidade do hidrodinamismo induzido por ondas. $\mathrm{O}$ conteúdo arenoso, de regiōes mais profundas, seria resultante da ausência de deposição de pelitos, em conseqüência da distância das fontes de sedimentos. Portanto, nāo é a estrutura sedimentar em si que limita o número de espécies, mas sim a sua instabilidade.

Os altos índices de diversidade específica na área de estudo, devem estar relacionados a outros fatores além daqueles relacionados à estrutura e textura do sedimento. Entre estes fatores se destacam a predação (Menge \& Sutherland, 1976), a estabilidade ambiental (Sanders, 1968; Grassle \& Sanders, 1973) e a competição por recursos (Dayton \& Hessler, 1972).

Huston (1979), congregou as várias hipóteses sobre fatores diversificadores em um único modelo baseado (1) na taxa de crescimento das populaçōes e, (2) na freqüência das perturbaçōes que afetam o ecossistema (predaçāo, perturbaçōes físicas e outros fatores).

A ausência de controle experimental de algumas variáveis relacionadas ao modelo, impede a aplicabilidade do modelo de Huston (op. cit.) para a área estudada. Não obstante, variaçōes da diversidade e eqüitatividade ao longo do gradiente costa-mar parecem estar relacionadas a diferenças quanto à freqüência de perturbaçōes. Nas estaçōes mais rasas da plataforma externa (isóbata de $20 \mathrm{~m}$ ), a freqüência e intensidade de perturbaçōes, como revolvimento do fundo por ondas, predaçāo por peixes e caranguejos (Petti, 1990; Soares $e t$ al., 1989a,b; Amaral et al., 1992), sedimentação e influência antrópica (descarga de poluentes, efeito mecânico de redes de arrasto, p. ex.) deve ser maior, inibindo o estabelecimento de uma série de espécies e permitindo a proliferaçāo de espécies mais oportunistas, elevando a dominância e condicionando, portanto, as baixas diversidades e eqüitatividades encontradas nesta faixa de profundidade.

Na plataforma externa, onde a estabilidade é maior, as altas diversidades estão relacionadas à ausência de dominância e à maior riqueza das areias finas.

$\mathrm{Na}$ faixa intermediária (entre 35 e $50 \mathrm{~m}$ ), o padrāo de incidência de ondas de grande comprimento é mais sazonal, enquanto os demais fatores perturbadores apresentam uma variabilidade de freqüência e intensidade muito grande, originando padrōes de diversidade mais complexos com verdadeiros mosaicos espaciais e temporais (Johnson, 1972).

\section{Conclusões}

Pode se concluir que a estrutura sedimentar, ou mesmo - hidrodinamismo que condiciona a deposição de diferentes tipos sedimęntológicos, têm um papel importante na determinação dos padrōes de densidade e diversidade específica. Outros fatores físicos e biológicos, cujos papéis são de difícil avaliaçāo num trabalho de cunho descritivo, devem ser responsáveis por aqueles padrōes de densidade e diversidade que independem da estrutura sedimentar.

\section{Agradecimentos}

O presente trabalho é parte integrante da dissertação de mestrado do autor, realizada com auxilio do CNPq e da CAPES, na forma de bolsa de estudos, e da CIRM (Comissão Interministerial para os Recursos do Mar) na forma de financiamento do Projeto: "Utilização Racional do Ecossistema Costeiro da Regiāo Tropical Brasileira: Estado de São Paulo" realizado pelo IOUSP. O autor é grato à Dra Ana Maria Pires-Vanin, coordenadora do subprojeto Bentos, pela cessão do material estudado; ao Dr Edmundo Ferraz Nonato pela orientação no decorrer do trabalho e ao Dr Michel Michaelovith de Mahiques e Dra Terezinha Monteiro Absher, pela leitura crítica do manuscrito. 


\section{Referências bibliográficas}

AMARAL, A. C. Z. 1980. Anelídeos poliquetos do infralitoral em duas enseadas da regiâo de Ubatuba. II. Aspectos ecológicos. Bolm Inst. oceanogr., S Paulo, 29(1):69-87.

; NONATO, E. F. \& PETTI, M. A. V. 1992. Contribution of the polychaetous annelids to the diet of some Brazilian fish. In: INTERNATIONAL POLYCHAETE CONFERENCE, 4., Angers, 1992. Abstracts.

CASTRO FILHO, B. M.; MIRANDA, L. B. \& MIYAO, S. Y. 1987. Condiçōes hidrográficas na plataforma continental ao largo de Ubatuba: variaçōes sazonais e em média escala. Bolm Inst. oceanogr., S Paulo, 35(2):135-151.

DAYTON, P. K. \& HESSLER, R. R. 1972. Role of biological disturbance in maintaining diversity in the deep sea. Deep-Sea Res., 19:199-208.

EMÍLSSON, I. 1959. Alguns aspectos fisicos e quimicos das águas marinhas brasileiras. Ciênc. Cult., S Paulo, 11(2):44-54.

FOLK, R. L. \& WARD, W. C. 1957. Brazos River bar: a study in the significance of grain size parameters. J. sedim. Petrology, 27:3-26.

FRESI, E.; GAMBI, M. C.; FOCARDI, S.; BARGAGLI, R.; BALDI, F. \& FALLIACI, L. 1983. Benthic community and sediment types: a structural analysis. Mar. Ecol., 4(2):101-121.

FURTADO, V. V. \& MAHIQUES, M. M. de 1990. Distribuição de sedimentos em regiōes costeiras e plataforma continental norte do Estado de São Paulo. In: SIMPÓSIO DE ECOSSISTEMAS DA COSTA SUL E SUDESTE BRASILEIRA: ESTRUTURA, FUNCĀO E MANEJO, 2, Águas de Lindóia, 1990. Sāo Paulo, Academia de Ciências do Estado de Sāo Paulo. v.1, p. 20-29.

GAMBI, M. C. \& FRESI, E. 1981. Ecology of softbottom macrobenthos along the coast of southern Tuscany (Parco Naturale della Maremma). Rapp. P.-v. Réun. Commn int. Expl. scient. Mer médit., 27(2):123-125.

GRASSLE, J. F. \& SANDERS, H. L. 1973. Life histories and the role of disturbance. Deep-Sea Res., 20:643-659.

GRAY, J. S. 1981. The ecology of marine sediments. Cambridge, Cambridge University Press. 185p.

GROSS, M. E. 1971. Carbon determination. In: Carver, R.E., ed. Producers in sedimentary petrology. New York, Wiley-Interscience. p.573-596.
HYLAND, J.; BAPTISTE, E; CAMPBELL, J.; KENNEDY, J.; KROPP, R. \& WILLIAMS, S. 1991. Macroinfaunal communities of the Santa Maria Basin on the outer continental shelf and slope. Mar. Ecol.- Prog. Ser., 78:147-161.

HUSTON, M. 1979. A general hypotesis of species diversity. Am. Naturalist, 113:81-101.

KNOX, G. A. 1977. The role of polychaetes in benthic soft-bottom communities. In: Reish, D. \& Fauchald, K., eds Essays on polychaetous annelids in memory of Dr. Olga Hartman. Los Angeles, Allan Hancock Foundation. p. 547-604.

JOHNSON, R. G. 1972. Conceptual models of benthic marine communities. In: Schopf, T. J. M., ed. Models in paleobiology. San Francisco, Freeman, Cooper. p. 149-159.

LANA, P. C. 1981. Padrōes de distribuiçāo e diversidade específica de anelídeos poliquetos na regiāo de Ubatuba, Estado de São Paulo. Dissertação de mestrado. Universidade de São Paulo, Instituto Oceanográfico. 111p.

1982. Um novo índice para avaliar a heterogeneidade de sedimentos não consolidados. Arq. Biol. Tecnol. 25(3/4):357-360.

LEGENDRE, L. \& LEGENDRE, P. 1983. Numerical ecology. New York, Elsevier. 419p.

MASSÉ, H. 1972. Quantitative investigations of sand-bottom macrofauna along the Mediterranean north-west coast. Mar. Biol., 15:209-220.

MATSUURA, Y. 1986. Contribuição ao estudo da estrutura oceanográfica da região sudeste entre Cabo Frio (RJ) e Cabo de Santa Marta Grande (SC). Ciênc. Cult., S Paulo, 38(8):1439-1450.

MENGE, B. A. \& SUTHERLAND, J. P. 1976. Species diversity gradientes: synthesis of the roles of predation, competition and temporal heterogeneity. Am. Naturalist, 110:351-369.

MORGADO, E. H. 1988. Anelídeos poliquetos do sublitoral da regiāo de Ubatuba-SP, compreendida entre as ilhas Anchieta e Vitória. Tese de doutorado. Universidade Estadual de Campinas, Instituto de Biologia. 181p.

PETTI, M. A. V. 1990. Hábitos alimentares dos crustáceos decápodos braquiúros e seu papel na rede trófica do infralitoral de Ubatuba (litoral norte do Estado de São Paulo, Brasil). Dissertação de mestrado. Universidade de São Paulo, Instituto Oceanográfico. $150 \mathrm{p}$. 
PICARD, J. 1965. Recherches qualitatives sur les biocenoses marines des substrats meubles dragables de la région marsillaise. Thèse. Faculté des Sciences. Université Aix Marseille. 325p.

PIELOU, E. C. 1975. Ecological diversity. New York, John Wiley. 165p.

PROBERT, P. K. 1984. Disturbance, sediment stability, and trophic structure of soft-bottom communities. J. mar. Res., 42:893-921.

RHOADS, D. C. \& GERMANO, J. D. 1982. Characterization of organism-sediment relations using sediment profile imaging: an efficient method of remote ecological monitoring of the seafloor (Remots TM system). Mar. Ecol.-Prog. Ser., 8:115-128.

SANDERS, H. L. 1968. Marine benthic diversity: a comparative study. Am. Naturalist, 102:243-282.

SHANNON, C. E. \& WEAVER, W. W. 1963. The mathematical theory of communications. Urbana, University of Illinois Press. 117p.

SOARES, L. S. H.; ROSSI-WONGTSCHOWSKI, C. L. D. B.; REYNA, M. J.; GASALLA, M. A.; ARRASA, M. V.;RIOS, M.A.T.1989a. Ecologia trófica da ictiofauna do sistema costeiro do litoral de Ubatuba, São Paulo, Brasil. I. Sciaenidae. In: SIMPÓSIO SOBRE OCEANOGRAFIA, 1., São Paulo, 1989. Resumos. São Paulo, Instituto Oceanográfico. p. 29.
SOARES, L. S. H.; ROSSI-WONGTSCHOWSKI, C. L. D. B.; ALVARES, L. M. C; APELBAUM, R.; MUTTO, E. Y. 1989b. Ecologia trófica da ictiofauna do sistema costeiro do litoral de Ubatuba, São Paulo, Brasil. III. Chondrichthyes. In: SIMPÓSIO SOBRE OCEANOGRAFIA, 1., São Paulo, 1989. Resumos. São Paulo, Instituto Oceanografico. p. 30.

STRICKLAND, J. D. \& PARSONS, T. R. 1968. A practical handbook of seawater analysis. Bull. Fish. Res. Bd Can., (167):1-311.

SUGUIO, K. 1973. Introdução à sedimentologia. São Paulo, Blücher/EDUSP. 312p.

TESSLER. M. G. 1988. Dinâmica sedimentar quaternária no litoral sul paulista. Tese de doutorado. Universidade de São Paulo, Instituto de Geociências. 277p.

VALIELA, I. 1984. Marine ecological processes. New York, Springer-Verlag. 546p.

WESTON, D. P. 1988. Macrobenthos-sediment relationships on the continental shelf off Cape Hatteras, North Carolina. Cont. Shelf Res., 8(3):267-286.

(Manuscrito recebido 9 setembro 1992; revisto 25 fevereiro 1993; aceito 26 de fevereiro 1993) 